\title{
AN IMPROVEMENT OF SOME INEQUALITIES SIMILAR TO HILBERT'S INEQUALITY
}

\author{
YOUNG-HO KIM
}

(Received 7 March 2001)

\begin{abstract}
We give an improvement of some inequalities similar to Hilbert's inequality involving series of nonnegative terms. The integral analogies of the main results are also given.
\end{abstract}

2000 Mathematics Subject Classification. 26D15.

1. Introduction. It is well known that the following Hilbert's double series inequality (see [3, page 226]) plays an important role in many branches of mathematics.

THEOREM 1.1. If $p>1, p^{\prime}=p /(p-1)$ and $\sum a_{m}^{p} \leq A, \sum b_{n}^{p^{\prime}} \leq B$, the summations running 1 to $\infty$, then

$$
\sum \sum \frac{a_{m} b_{n}}{m+n}<\frac{\pi}{\sin (\pi / p)} A^{1 / p} B^{1 / p^{\prime}}
$$

unless all the sequence $\left\{a_{m}\right\}$ or $\left\{b_{n}\right\}$ is null.

The integral analogue of Hilbert's inequality can be stated as follows (see [3, page 226]).

THEOREM 1.2. If $p>1, p^{\prime}=p /(p-1)$ and

$$
\int_{0}^{\infty} f^{p}(x) d x \leq F, \quad \int_{0}^{\infty} g^{p^{\prime}}(y) d y \leq G,
$$

then

$$
\int_{0}^{\infty} \int_{0}^{\infty} \frac{f(x) g(y)}{x+y} d x d y<\frac{\pi}{\sin (\pi / p)} F^{1 / p} G^{1 / p^{\prime}}
$$

unless $f \equiv 0$ or $g \equiv 0$.

These two theorems were studied extensively and numerous variants, generalizations, and extensions appeared in the literature, see [1, 2, 3, 5, 6, 9] and the references therein.

Recently, Pachpatte [9] gave new inequalities similar to Hilbert's inequalities given in the above theorems, involving a series of nonnegative terms as follows.

THEOREM 1.3. Let $p \geq 1, q \geq 1$, and let $\left\{a_{m}\right\}$ and $\left\{b_{n}\right\}$ be two nonnegative sequences of real numbers defined for $m=1,2, \ldots, k$ and $n=1,2, \ldots, r$, where $k, r$ are the natural 
numbers and define $A_{m}=\sum_{s=1}^{m} a_{s}, B_{n}=\sum_{t=1}^{n} b_{t}$. Then

$$
\begin{aligned}
\sum_{m=1}^{k} \sum_{n=1}^{r} \frac{A_{m}^{p} B_{n}^{q}}{m+n} \leq & C(p, q, k, r)\left(\sum_{m=1}^{k}(k-m+1)\left(A_{m}^{p-1} a_{m}\right)^{2}\right)^{1 / 2} \\
& \times\left(\sum_{n=1}^{r}(r-n+1)\left(B_{n}^{q-1} b_{n}\right)^{2}\right)^{1 / 2}
\end{aligned}
$$

unless $\left\{a_{m}\right\}$ or $\left\{b_{n}\right\}$ is null, where

$$
C(p, q, k, r)=\frac{1}{2} p q \sqrt{k r} .
$$

An integral analogue of Theorem 1.3 is given in the following theorem.

THEOREM 1.4. Let $p \geq 1, q \geq 1$ and $f(\sigma) \geq 0, g(\tau) \geq 0$ for $\sigma \in(0, x), \tau \in(0, y)$, where $x, y$ are positive real numbers and define $F(s)=\int_{0}^{s} f(\sigma) d \sigma$ and $G(t)=\int_{0}^{t} g(\tau) d \tau$, for $s \in(0, x), t \in(0, y)$. Then

$$
\begin{aligned}
\int_{0}^{x} \int_{0}^{y} \frac{F^{p}(s) G^{q}(t)}{s+t} d s d t \leq & D(p, q, x, y)\left(\int_{0}^{x}(x-s)\left(F^{p-1}(s) f(s)\right)^{2} d s\right)^{1 / 2} \\
& \times\left(\int_{0}^{y}(y-t)\left(G^{q-1}(t) g(t)\right)^{2} d t\right)^{1 / 2}
\end{aligned}
$$

unless $f \equiv 0$ or $g \equiv 0$, where

$$
D(p, q, x, y)=\frac{1}{2} p q \sqrt{x y} .
$$

In this paper, we give an improvement of the inequalities given in Theorems 1.3 and 1.4 similar to Hilbert's double series inequality and its integral analogue, involving a series of nonnegative terms. In addition, we obtain some new Hilbert type inequalities. These inequalities improve the results obtained by Pachpatte [9].

2. Main results. Our main results are given in the following theorems.

THEOREM 2.1. Let $p \geq 1, q \geq 1,0<\alpha$, and let $\left\{a_{m}\right\}$ and $\left\{b_{n}\right\}$ be two nonnegative sequences of real numbers defined for $m=1,2, \ldots, k$ and $n=1,2, \ldots, r$, where $k, r$ are the natural numbers and define $A_{m}=\sum_{s=1}^{m} a_{s}, B_{n}=\sum_{t=1}^{n} b_{t}$. Then

$$
\begin{aligned}
\sum_{m=1}^{k} \sum_{n=1}^{r} \frac{A_{m}^{p} B_{n}^{q}}{\left(m^{\alpha}+n^{\alpha}\right)^{1 / \alpha}} & \leq(p, q, k, r ; \alpha)\left(\sum_{m=1}^{k}(k-m+1)\left(A_{m}^{p-1} a_{m}\right)^{2}\right)^{1 / 2} \\
& \times\left(\sum_{n=1}^{r}(r-n+1)\left(B_{n}^{q-1} b_{n}\right)^{2}\right)^{1 / 2},
\end{aligned}
$$

unless $\left\{a_{m}\right\}$ or $\left\{b_{n}\right\}$ is null, where

$$
C(p, q, k, r ; \alpha)=\left(\frac{1}{2}\right)^{1 / \alpha} p q \sqrt{k r} .
$$


Proof. By using the following inequality (see [1, 7]),

$$
\left(\sum_{m=1}^{n} z_{m}\right)^{\beta} \leq \beta \sum_{m=1}^{n} z_{m}\left(\sum_{k=1}^{m} z_{k}\right)^{\beta-1}
$$

where $\beta \geq 1$ is a constant and $z_{m} \geq 0,(m=1,2, \ldots)$, it is easy to observe that

$$
A_{m}^{p} \leq p \sum_{s=1}^{m} a_{s} A_{s}^{p-1}, \quad m=1,2, \ldots, k, \quad B_{n}^{q} \leq q \sum_{t=1}^{n} a_{t} B_{t}^{q-1}, \quad n=1,2, \ldots, r .
$$

From (2.4) and using the Schwarz inequality and the elementary inequality

$$
\left(\prod_{i=1}^{n} a_{i}\right)^{1 / n} \leq\left(\sum_{i=1}^{n} \frac{a_{i}^{\alpha}}{n}\right)^{1 / \alpha}, \quad 0<\alpha, \text { (see [4]), }
$$

(for $a_{i}, i=1,2, \ldots, n$, nonnegative real numbers) we observe that

$$
\begin{aligned}
A_{m}^{p} B_{n}^{q} & \leq p q\left(\sum_{s=1}^{m} a_{s} A_{s}^{p-1}\right)\left(\sum_{t=1}^{n} a_{t} B_{t}^{q-1}\right) \\
& \leq p q(m)^{1 / 2}\left(\sum_{s=1}^{m}\left(a_{s} A_{s}^{p-1}\right)^{2}\right)^{1 / 2}(n)^{1 / 2}\left(\sum_{t=1}^{n}\left(a_{t} B_{t}^{q-1}\right)^{2}\right)^{1 / 2} \\
& \leq p q\left(\frac{m^{\alpha}+n^{\alpha}}{2}\right)^{1 / \alpha}\left(\sum_{s=1}^{m}\left(a_{s} A_{s}^{p-1}\right)^{2}\right)^{1 / 2}\left(\sum_{t=1}^{n}\left(a_{t} B_{t}^{q-1}\right)^{2}\right)^{1 / 2} .
\end{aligned}
$$

Dividing both sides of (2.6) by $\left(m^{\alpha}+n^{\alpha}\right)^{1 / \alpha}$, and then taking the sum over $n$ from 1 to $r$ first and then the sum over $m$ from 1 to $k$ and using the Schwarz inequality and then interchanging the order of the summations (see $[7,8]$ ) we observe that

$$
\begin{aligned}
\sum_{m=1}^{k} & \sum_{n=1}^{r} \frac{A_{m}^{p} B_{n}^{q}}{\left(m^{\alpha}+n^{\alpha}\right)^{1 / \alpha}} \\
& \leq p q\left(\frac{1}{2}\right)^{1 / \alpha}\left\{\sum_{m=1}^{k}\left(\sum_{s=1}^{m}\left(a_{s} A_{s}^{p-1}\right)^{2}\right)^{1 / 2}\right\}\left\{\sum_{n=1}^{r}\left(\sum_{t=1}^{n}\left(b_{t} B_{t}^{q-1}\right)^{2}\right)^{1 / 2}\right\} \\
& \leq p q\left(\frac{1}{2}\right)^{1 / \alpha}(k)^{1 / 2}\left\{\sum_{m=1}^{k}\left(\sum_{s=1}^{m}\left(a_{s} A_{s}^{p-1}\right)^{2}\right)\right\}^{1 / 2}(r)^{1 / 2}\left\{\sum_{n=1}^{r}\left(\sum_{t=1}^{n}\left(b_{t} B_{t}^{q-1}\right)^{2}\right)\right\}^{1 / 2} \\
& =p q \sqrt{k r}\left(\frac{1}{2}\right)^{1 / \alpha}\left\{\sum_{s=1}^{k}\left(a_{s} A_{s}^{p-1}\right)^{2}\left(\sum_{m=s}^{k} 1\right)\right\}^{1 / 2}\left\{\sum_{t=1}^{r}\left(b_{t} B_{t}^{q-1}\right)^{2}\left(\sum_{n=t}^{r} 1\right)\right\}^{1 / 2} \\
& =C(p, q, k, r ; \alpha)\left\{\sum_{s=1}^{k}\left(a_{s} A_{s}^{p-1}\right)^{2}(k-s+1)\right\}^{1 / 2}\left\{\sum_{t=1}^{r}\left(b_{t} B_{t}^{q-1}\right)^{2}(r-t+1)\right\}^{1 / 2} \\
& =C(p, q, k, r ; \alpha)\left\{\sum_{m=1}^{k}(k-m+1)\left(a_{m} A_{m}^{p-1}\right)^{2}\right\}^{1 / 2}\left\{\sum_{n=1}^{r}(r-n+1)\left(b_{n} B_{n}^{q-1}\right)^{2}\right\}^{1 / 2} .
\end{aligned}
$$

This completes the proof. 
REMARK 2.2. In Theorem 2.1, setting $\alpha \equiv 1$, we have Theorem 1.3. If we take $p=$ $q=1$ in Theorem 2.1, then the inequality of the result of Theorem 2.1 reduces to the following inequality:

$$
\begin{aligned}
\sum_{m=1}^{k} \sum_{n=1}^{r} \frac{A_{m} B_{n}}{\left(m^{\alpha}+n^{\alpha}\right)^{1 / \alpha}} \leq & C(1,1, k, r ; \alpha)\left(\sum_{m=1}^{k}(k-m+1)\left(a_{m}\right)^{2}\right)^{1 / 2} \\
& \times\left(\sum_{n=1}^{r}(r-n+1)\left(b_{n}\right)^{2}\right)^{1 / 2}
\end{aligned}
$$

where $C(1,1, k, r ; \alpha)$ is obtained by taking $p=q=1$ in (2.2).

Our next result deals with further generalization of the inequality obtained in Remark 2.2.

TheOrem 2.3. Let $\left\{a_{m}\right\},\left\{b_{n}\right\}, A_{m}$, and $B_{n}$ be as defined in Theorem 2.1. Let $\left\{p_{m}\right\}$ and $\left\{q_{n}\right\}$ be two nonnegative sequences for $m=1,2, \ldots, k$ and $n=1,2, \ldots, r$, and define $P_{m}=\sum_{s=1}^{m} p_{s}, Q_{n}=\sum_{t=1}^{n} q_{t}$. Let $\phi$ and $\psi$ be two real-valued, nonnegative, convex, and submultiplicative functions defined on $\mathbb{R}_{+}=[0, \infty)$. Then

$$
\begin{aligned}
\sum_{m=1}^{k} \sum_{n=1}^{r} \frac{\phi\left(A_{m}\right) \psi\left(B_{n}\right)}{\left(m^{\alpha}+n^{\alpha}\right)^{1 / \alpha}} \leq & M(k, r ; \alpha)\left(\sum_{m=1}^{k}(k-m+1)\left[p_{m} \phi\left(\frac{a_{m}}{p_{m}}\right)\right]^{2}\right)^{1 / 2} \\
& \times\left(\sum_{n=1}^{r}(r-n+1)\left[q_{n} \phi\left(\frac{b_{n}}{a_{n}}\right)\right]^{2}\right)^{1 / 2},
\end{aligned}
$$

where

$$
M(k, r ; \alpha)=\left(\frac{1}{2}\right)^{1 / \alpha}\left(\sum_{m=1}^{k}\left[\frac{\phi\left(P_{m}\right)}{P_{m}}\right]^{2}\right)^{1 / 2}\left(\sum_{n=1}^{r}\left[\frac{\psi\left(Q_{n}\right)}{Q_{n}}\right]^{2}\right)^{1 / 2}
$$

Proof. From the hypotheses of $\phi$ and $\psi$ and by using Jensen's inequality and the Schwarz inequality (see [5]), it is easy to observe that

$$
\begin{aligned}
\phi\left(A_{m}\right) & =\phi\left(\frac{P_{m} \sum_{s=1}^{m} p_{s} a_{s} / p_{s}}{\sum_{s=1}^{m} p_{s}}\right) \leq \phi\left(P_{m}\right) \phi\left(\frac{\sum_{s=1}^{m} p_{s} a_{s} / p_{s}}{\sum_{s=1}^{m} p_{s}}\right) \\
& \leq \frac{\phi\left(P_{m}\right)}{P_{m}} \sum_{s=1}^{m} p_{s} \phi\left(\frac{a_{s}}{p_{s}}\right) \leq \frac{\phi\left(P_{m}\right)}{P_{m}}(m)^{1 / 2}\left(\sum_{s=1}^{m}\left[p_{s} \phi\left(\frac{a_{s}}{p_{s}}\right)\right]^{2}\right)^{1 / 2},
\end{aligned}
$$

and similarly,

$$
\psi\left(B_{n}\right) \leq \frac{\psi\left(Q_{n}\right)}{Q_{n}}(n)^{1 / 2}\left(\sum_{t=1}^{n}\left[q_{t} \psi\left(\frac{b_{t}}{q_{t}}\right)\right]^{2}\right)^{1 / 2} .
$$

From (2.11) and (2.12) and using the elementary inequality

$$
\left(\prod_{i=1}^{n} a_{i}\right)^{1 / n} \leq\left(\sum_{i=1}^{n} \frac{a_{i}^{\alpha}}{n}\right)^{1 / \alpha}, \quad 0<\alpha
$$


(for $a_{i}, i=1,2, \ldots, n$, nonnegative real numbers) we observe that

$$
\begin{aligned}
\phi\left(A_{m}\right) \psi\left(B_{n}\right) \leq & \left(\frac{m^{\alpha}+n^{\alpha}}{2}\right)^{1 / \alpha}\left\{\frac{\phi\left(P_{m}\right)}{P_{m}}\left(\sum_{s=1}^{m}\left[p_{s} \phi\left(\frac{a_{s}}{p_{s}}\right)\right]^{2}\right)^{1 / 2}\right\} \\
& \times\left\{\frac{\psi\left(Q_{n}\right)}{Q_{n}}\left(\sum_{t=1}^{n}\left[q_{t} \psi\left(\frac{b_{t}}{q_{t}}\right)\right]^{2}\right)^{1 / 2}\right\} .
\end{aligned}
$$

Dividing both sides of the above inequality by $\left(m^{\alpha}+n^{\alpha}\right)^{1 / \alpha}$, and then taking the sum over $n$ from 1 to $r$ first and then the sum over $m$ from 1 to $k$ and using the Schwarz inequality and then interchanging the order of the summations we observe that

$$
\begin{aligned}
\sum_{m=1}^{k} \sum_{n=1}^{r} & \frac{\phi\left(A_{m}\right) \psi\left(B_{n}\right)}{\left(m^{\alpha}+n^{\alpha}\right)^{1 / \alpha}} \\
\leq & \left(\frac{1}{2}\right)^{1 / \alpha}\left[\sum_{m=1}^{k}\left\{\frac{\phi\left(P_{m}\right)}{P_{m}}\left(\sum_{s=1}^{m}\left[p_{s} \phi\left(\frac{a_{s}}{p_{s}}\right)\right]^{2}\right)^{1 / 2}\right\}\right] \\
& \times\left[\sum_{n=1}^{r}\left\{\frac{\psi\left(Q_{n}\right)}{Q_{n}}\left(\sum_{t=1}^{n}\left[q_{t} \psi\left(\frac{b_{t}}{q_{t}}\right)\right]^{2}\right)^{1 / 2}\right\}\right] \\
\leq & \left(\frac{1}{2}\right)^{1 / \alpha}\left(\sum_{m=1}^{k}\left[\frac{\phi\left(P_{m}\right)}{P_{m}}\right]^{2}\right)^{1 / 2}\left(\sum_{m=1}^{k}\left(\sum_{s=1}^{m}\left[p_{s} \phi\left(\frac{a_{s}}{p_{s}}\right)\right]^{2}\right)\right)^{1 / 2} \\
& \times\left(\sum_{n=1}^{r}\left[\frac{\psi\left(Q_{n}\right)}{Q_{n}}\right]^{2}\right)^{1 / 2}\left(\sum_{n=1}^{r}\left(\sum_{t=1}^{n}\left[q_{t} \psi\left(\frac{b_{t}}{q_{t}}\right)\right]^{2}\right)\right)^{1 / 2} \\
= & M(k, r ; \alpha)\left(\sum_{s=1}^{k}\left[p_{s} \phi\left(\frac{a_{s}}{p_{s}}\right)\right]^{2}\left(\sum_{m=s}^{k} 1\right)\right)^{1 / 2}\left(\sum_{t=1}^{r}\left[q_{t} \psi\left(\frac{b_{t}}{q_{t}}\right)\right]^{2}\left(\sum_{n=t}^{r} 1\right)\right)^{1 / 2} \\
= & M(k, r ; \alpha)\left(\sum_{s=1}^{k}\left[p_{s} \phi\left(\frac{a_{s}}{p_{s}}\right)\right]^{2}(k-s+1)\right)^{1 / 2}\left(\sum_{t=1}^{r}\left[q_{t} \psi\left(\frac{b_{t}}{q_{t}}\right)\right]^{2}(r-t+1)\right)^{1 / 2} \\
= & M(k, r ; \alpha)\left(\sum_{m=1}^{k}(k-m+1)\left[p_{m} \phi\left(\frac{a_{m}}{p_{m}}\right)\right]^{2}\right)^{1 / 2}\left(\sum_{n=1}^{r}(r-n+1)\left[q_{n} \psi\left(\frac{b_{n}}{q_{n}}\right)\right]^{2}\right)^{1 / 2} .
\end{aligned}
$$

The proof is complete.

REMARK 2.4. By applying the elementary inequality (see [4])

$$
\left(\prod_{i=1}^{n} a_{i}\right)^{1 / n} \leq\left(\sum_{i=1}^{n} \frac{a_{i}^{\gamma}}{n}\right)^{1 / \gamma}, \quad 0<\gamma,
$$

(for $a_{i}, i=1,2, \ldots, n$, nonnegative real numbers) on the right sides of the inequalities 
in Theorems 2.1 and 2.3, we get, respectively, the following inequalities:

$$
\begin{aligned}
& \sum_{m=1}^{k} \sum_{n=1}^{r} \frac{A_{m}^{p} B_{n}^{q}}{\left(m^{\alpha}+n^{\alpha}\right)^{1 / \alpha}} \\
& \quad \leq C_{1}\left(\left(\sum_{m=1}^{k}(k-m+1)\left(A_{m}^{p-1} a_{m}\right)^{2}\right)^{\gamma}+\left(\sum_{n=1}^{r}(r-n+1)\left(B_{n}^{q-1} b_{m}\right)^{2}\right)^{\gamma}\right)^{1 / \gamma},
\end{aligned}
$$

where $C_{1}=(1 / 2)^{1 / \gamma} C(p, q, k, r ; \alpha)$, and

$$
\begin{aligned}
& \sum_{m=1}^{k} \sum_{n=1}^{r} \frac{\phi\left(A_{m}\right) \psi\left(B_{n}\right)}{\left(m^{\alpha}+n^{\alpha}\right)^{1 / \alpha}} \\
& \quad \leq M_{1}\left(\left(\sum_{m=1}^{k}(k-m+1)\left[p_{m} \phi\left(\frac{a_{m}}{p_{m}}\right)\right]^{2}\right)^{\gamma}+\left(\sum_{n=1}^{r}(r-n+1)\left[q_{n} \psi\left(\frac{b_{n}}{q_{n}}\right)\right]^{2}\right)^{\gamma}\right)^{1 / \gamma},
\end{aligned}
$$

where $M_{1}=(1 / 2)^{1 / \gamma} M(k, r ; \alpha)$, which we believe are new to the literature.

The following theorems deal with slight variants of (2.9) given in Theorem 2.3.

THEOREM 2.5. Let $\left\{a_{m}\right\}$ and $\left\{b_{n}\right\}$ be as defined in Theorem 2.1, and define $A_{m}=$ $1 / m \sum_{s=1}^{m} a_{s}$ and $B_{n}=1 / n \sum_{t=1}^{n} b_{t}$, for $m=1,2, \ldots, k$ and $n=1,2, \ldots, r$, where $k, r$ are the natural numbers. Let $\phi$ and $\psi$ be two real-valued, nonnegative, and convex functions defined on $\mathbb{R}_{+}=[0, \infty)$. Then

$$
\begin{aligned}
\sum_{m=1}^{k} \sum_{n=1}^{r} \frac{m n}{\left(m^{\alpha}+n^{\alpha}\right)^{1 / \alpha}} \phi\left(A_{m}\right) \psi\left(B_{n}\right) \leq & C(1,1, k, r ; \alpha)\left(\sum_{m=1}^{k}(k-m+1)\left[\phi\left(a_{m}\right)\right]^{2}\right)^{1 / 2} \\
& \times\left(\sum_{n=1}^{r}(r-n+1)\left[\psi\left(b_{n}\right)\right]^{2}\right)^{1 / 2}
\end{aligned}
$$

where $C(1,1, k, r)$ is defined by taking $p=q=1$ in (2.2).

Proof. From the hypotheses and by using Jensen's inequality and the Schwarz inequality, it is easy to observe that

$$
\begin{aligned}
& \phi\left(A_{m}\right)=\phi\left(\frac{1}{m} \sum_{s=1}^{m} a_{s}\right) \leq \frac{1}{m} \sum_{s=1}^{m} \phi\left(a_{s}\right) \leq \frac{1}{m}(m)^{1 / 2}\left(\sum_{s=1}^{m}\left[\phi\left(a_{s}\right)\right]^{2}\right)^{1 / 2}, \\
& \psi\left(B_{m}\right)=\psi\left(\frac{1}{n} \sum_{t=1}^{n} b_{t}\right) \leq \frac{1}{n} \sum_{t=1}^{n} \psi\left(b_{t}\right) \leq \frac{1}{n}(n)^{1 / 2}\left(\sum_{t=1}^{n}\left[\psi\left(b_{t}\right)\right]^{2}\right)^{1 / 2} .
\end{aligned}
$$

The rest of the proof can be completed by following the same steps as in the proofs of Theorems 2.1 and 2.3 with suitable changes and hence we omit the details.

TheOREM 2.6. Let $\left\{a_{m}\right\},\left\{b_{n}\right\},\left\{p_{m}\right\},\left\{q_{n}\right\}, P_{m}$, and $Q_{n}$ be as in Theorem 2.3, and define $A_{m}=1 / P_{m} \sum_{s=1}^{m} p_{s} a_{s}$ and $B_{n}=1 / Q_{n} \sum_{t=1}^{n} q_{t} b_{t}$, for $m=1,2, \ldots, k$ and 
$n=1,2, \ldots, r$, where $k, r$ are the natural numbers. Let $\phi$ and $\psi$ be as defined in Theorem 2.5. Then

$$
\begin{aligned}
\sum_{m=1}^{k} \sum_{n=1}^{r} \frac{P_{m} Q_{n} \phi\left(A_{m}\right) \psi\left(B_{n}\right)}{\left(m^{\alpha}+n^{\alpha}\right)^{1 / \alpha}} \leq & C(1,1, k, r ; \alpha)\left(\sum_{m=1}^{k}(k-m+1)\left[p_{s} \phi\left(a_{s}\right)\right]^{2}\right)^{1 / 2} \\
& \times\left(\sum_{n=1}^{r}(r-n+1)\left[q_{t} \psi\left(b_{t}\right)\right]^{2}\right)^{1 / 2},
\end{aligned}
$$

where $C(1,1, k, r ; \alpha)$ is defined by taking $p=q=1$ in (2.2).

Proof. From the hypotheses and by using Jensen's inequality and the Schwarz inequality, it is easy to observe that

$$
\begin{gathered}
\phi\left(A_{m}\right)=\phi\left(\frac{1}{P_{m}} \sum_{s=1}^{m} p_{s} a_{s}\right) \leq \frac{1}{P_{m}} \sum_{s=1}^{m} p_{s} \phi\left(a_{s}\right) \leq \frac{1}{P_{m}}(m)^{1 / 2}\left(\sum_{s=1}^{m} p_{s}\left[\phi\left(a_{s}\right)\right]^{2}\right)^{1 / 2}, \\
\phi\left(B_{n}\right)=\psi\left(\frac{1}{Q_{n}} \sum_{t=1}^{n} q_{t} b_{t}\right) \leq \frac{1}{Q_{n}} \sum_{t=1}^{n} q_{t} \psi\left(b_{t}\right) \leq \frac{1}{Q_{n}}(n)^{1 / 2}\left(\sum_{t=1}^{n}\left[q_{t} \psi\left(b_{t}\right)\right]^{2}\right)^{1 / 2} .
\end{gathered}
$$

The rest of the proof can be completed by following the same steps as in the proofs of Theorems 2.1 and 2.3 with suitable changes and hence we omit the details.

3. Integral analogues. In this section, we present the integral analogues of the inequalities given in Theorems 2.1, 2.3, 2.5, and 2.6, which in fact are motivated by the integral analogue of Hilbert's inequality given in Theorem 1.2.

An integral analogue of Theorem 2.1 is given in the following theorem.

THEOREM 3.1. Let $p \geq 1, q \geq 1,0<\alpha \leq 1$ and $f(\sigma) \geq 0, g(\tau) \geq 0$ for $\sigma \in(0, x)$, $\tau \in(0, y)$, where $x, y$ are positive real numbers, define $F(s)=\int_{0}^{s} f(\sigma) d \sigma$ and $G(t)=$ $\int_{0}^{t} g(\tau) d \tau$, for $s \in(0, x), t \in(0, y)$. Then

$$
\begin{aligned}
\int_{0}^{x} \int_{0}^{y} \frac{F^{p}(s) G^{q}(t)}{\left(s^{\alpha}+t^{\alpha}\right)^{1 / \alpha}} d s d t \leq & D(p, q, x, y ; \alpha)\left(\int_{0}^{x}(x-s)\left(F_{f}(s)\right)^{2} d s\right)^{1 / 2} \\
& \times\left(\int_{0}^{y}(y-t)\left(G_{g}(t)\right)^{2} d t\right)^{1 / 2}
\end{aligned}
$$

unless $f \equiv 0$ or $g \equiv 0$, where $F_{f}(s)=F^{p-1}(s) f(s), G_{g}(t)=G^{q-1}(t) g(t)$, and

$$
D(p, q, x, y ; \alpha)=\left(\frac{1}{2}\right)^{1 / \alpha} p q \sqrt{x y}
$$

Proof. From the hypotheses of $F(s)$ and $G(t)$, it is easy to observe that

$$
\begin{aligned}
& F^{p}(s)=p \int_{0}^{s} F^{p-1}(\sigma) f(\sigma) d \sigma, \quad s \in(0, x), \\
& G^{q}(t)=q \int_{0}^{t} G^{q-1}(\tau) g(\tau) d \tau, \quad t \in(0, y) .
\end{aligned}
$$


From (3.3) and using the Schwarz inequality and the elementary inequality

$$
\left(\prod_{i=1}^{n} a_{i}\right)^{1 / n} \leq\left(\sum_{i=1}^{n} \frac{a_{i}^{\alpha}}{n}\right)^{1 / \alpha}, \quad 0<\alpha
$$

(for $a_{i}, i=1,2, \ldots, n$, nonnegative real numbers) we observe that

$$
\begin{aligned}
F^{p}(s) G^{q}(t) & =p q\left(\int_{0}^{s} F^{p-1}(\sigma) f(\sigma) d \sigma\right)\left(\int_{0}^{t} G^{q-1}(\tau) g(\tau) d \tau\right) \\
& \leq p q(s)^{1 / 2}\left(\int_{0}^{s}\left(F^{p-1}(\sigma) f(\sigma)\right)^{2} d \sigma\right)^{1 / 2}(t)^{1 / 2}\left(\int_{0}^{t}\left(G^{q-1}(\tau) g(\tau)\right)^{2} d \tau\right)^{1 / 2} \\
& \leq p q\left(\frac{s^{\alpha}+t^{\alpha}}{2}\right)^{1 / \alpha}\left(\int_{0}^{s}\left(F^{p-1}(\sigma) f(\sigma)\right)^{2} d \sigma\right)^{1 / 2}\left(\int_{0}^{t}\left(G^{q-1}(\tau) g(\tau)\right)^{2} d \tau\right)^{1 / 2} .
\end{aligned}
$$

Dividing both sides of the above inequality by $\left(s^{\alpha}+t^{\alpha}\right)^{1 / \alpha}$, and then integrating over $t$ from 0 to $y$ first and then integrating the resulting inequality over $s$ from 0 to $x$ and using the Schwarz inequality we observe that

$$
\begin{aligned}
\int_{0}^{x} \int_{0}^{y} \frac{F^{p}(s) G^{q}(t)}{\left(s^{\alpha}+t^{\alpha}\right)^{1 / \alpha}} d s d t \\
\quad \leq p q\left(\frac{1}{2}\right)^{1 / \alpha}\left\{\int_{0}^{x}\left(\int_{0}^{s}\left(F_{f}(\sigma)\right)^{2} d \sigma\right)^{1 / 2} d t\right\}\left\{\int_{0}^{y}\left(\int_{0}^{t}\left(G_{g}(\tau)\right)^{2} d \tau\right)^{1 / 2} d t\right\} \\
\quad \leq p q\left(\frac{1}{2}\right)^{1 / \alpha}(x y)^{1 / 2}\left\{\int_{0}^{x}\left(\int_{0}^{s}\left(F_{f}(\sigma)\right)^{2} d \sigma\right) d s\right\}^{1 / 2}\left\{\int_{0}^{y}\left(\int_{0}^{t}\left(G_{g}(\tau)\right)^{2} d \tau\right) d t\right\}^{1 / 2} \\
\quad=D(p, q, x, y ; \alpha)\left(\int_{0}^{x}(x-s)\left(F_{f}(s)\right)^{2} d s\right)^{1 / 2}\left(\int_{0}^{y}(y-t)\left(G_{g}(t)\right)^{2} d t\right)^{1 / 2},
\end{aligned}
$$

where $F_{f}(\sigma)=F^{p-1}(\sigma) f(\sigma), G_{g}(\tau)=G^{q-1}(\tau) g(\tau)$. This completes the proof.

REMARK 3.2. In the special case when $p=q=1$, inequality (3.1) in Theorem 3.1 reduces to the following inequality:

$$
\begin{aligned}
& \int_{0}^{x} \int_{0}^{y} \frac{F(s) G(t)}{\left(s^{\alpha}+t^{\alpha}\right)^{1 / \alpha}} d s d t \\
& \quad=D(1,1, x, y ; \alpha)\left(\int_{0}^{x}(x-s) f^{2}(s) d s\right)^{1 / 2}\left(\int_{0}^{y}(y-t) g^{2}(t) d t\right)^{1 / 2},
\end{aligned}
$$

where $D(1,1, x, y ; \alpha)$ is obtained by taking $p=q=1$ in (3.2).

The integral analogues of the inequalities in Theorems 2.3, 2.5, and 2.6 are established in the following theorems. 
THEOREM 3.3. Let $f, g, F, G$ be as in Theorem 3.1. Let $p(\sigma)$ and $q(\tau)$ be two positive functions defined for $\sigma \in(0, x), \tau \in(0, y)$, and define $P(s)=\int_{0}^{s} p(\sigma) d \sigma$ and $Q(t)=$ $\int_{0}^{t} q(\tau) d \tau$, for $s \in(0, x), t \in(0, y)$, where $x, y$ are positive real numbers. Let $\phi$ and $\psi$ be as in Theorem 2.3. Then

$$
\begin{aligned}
\int_{0}^{x} \int_{0}^{y} \frac{\phi(F p(s)) \psi(G(t))}{\left(s^{\alpha}+t^{\alpha}\right)^{1 / \alpha}} d s d t \leq & L(x, y ; \alpha)\left(\int_{0}^{x}(x-s)\left[p(s) \phi\left(\frac{f(s)}{p(s)}\right)\right]^{2} d s\right)^{1 / 2} \\
& \times\left(\int_{0}^{y}(y-t)\left[q(t) \psi\left(\frac{g(t)}{q(t)}\right)\right]^{2} d t\right)^{1 / 2}
\end{aligned}
$$

where

$$
L(x, y ; \alpha)=\left(\frac{1}{2}\right)^{1 / \alpha}\left(\int_{0}^{x}\left[\frac{\phi(P(s))}{P(s)}\right]^{2} d s\right)^{1 / 2}\left(\int_{0}^{x}\left[\frac{\psi(Q(t))}{Q(t)}\right]^{2} d t\right)^{1 / 2} .
$$

Proof. From the hypotheses and by using Jensen's inequality and the Schwarz inequality, it is easy to observe that

$$
\begin{aligned}
\phi(F(s)) & =\phi\left(\frac{\left[P(s) \int_{0}^{s} P(s)(f(\sigma) / p(\sigma)) d \sigma\right]}{\left[\int_{0}^{s} p(\sigma) d \sigma\right]}\right) \\
& \leq \frac{\phi(P(s))}{P(s)} \int_{0}^{s} P(\sigma) \phi\left(\frac{f(\sigma)}{p(\sigma)}\right) d \sigma \\
& \leq \frac{\phi(P(s))}{P(s)}(s)^{1 / 2}\left(\int_{0}^{s}\left[P(\sigma) \phi\left(\frac{f(\sigma)}{p(\sigma)}\right)\right]^{2} d \sigma\right)^{1 / 2},
\end{aligned}
$$

and similarly,

$$
\psi(G(t)) \leq \frac{\psi(Q(t))}{Q(t)}(t)^{1 / 2}\left(\int_{0}^{t}\left[q(\tau) \psi\left(\frac{g(\tau)}{q(\tau)}\right)\right]^{2} d \tau\right)^{1 / 2}
$$

From (3.10) and (3.11) and using the elementary inequality

$$
\left(\prod_{i=1}^{n} a_{i}\right)^{1 / n} \leq\left(\sum_{i=1}^{n} \frac{a_{i}^{\alpha}}{n}\right)^{1 / \alpha}, \quad 0<\alpha
$$

(for $a_{i}, i=1,2, \ldots, n$, nonnegative real numbers) we observe that

$$
\begin{aligned}
\phi(F(s)) \psi(G(t)) \leq & \left(\frac{s^{\alpha}+t^{\alpha}}{2}\right)^{1 / \alpha}\left\{\frac{\phi(P(s))}{P(s)}(s)^{1 / 2}\left(\int_{0}^{s}\left[P(\sigma) \phi\left(\frac{f(\sigma)}{p(\sigma)}\right)\right]^{2} d \sigma\right)^{1 / 2}\right\} \\
& \times\left\{\frac{\psi(Q(t))}{Q(t)}(t)^{1 / 2}\left(\int_{0}^{t}\left[q(\tau) \psi\left(\frac{g(\tau)}{q(\tau)}\right)\right]^{2} d \tau\right)^{1 / 2}\right\} .
\end{aligned}
$$


The rest of the proof can be completed by following the same steps as in the proof of Theorem 3.1 and closely looking at the proof of Theorem 2.3, and hence we omit the details.

THEOREM 3.4. Let $f, g$, be as in Theorem 3.1, and define $F(s)=\int_{0}^{s} f(\sigma) d \sigma$ and $G(t)=\int_{0}^{t} g(\tau) d \tau$, for $s \in(0, x), t \in(0, y)$, where $x, y$ are positive real numbers. Let $\phi$ and $\psi$ be as in Theorem 2.5. Then

$$
\begin{aligned}
& \int_{0}^{x} \int_{0}^{y} \frac{s t}{\left(s^{\alpha}+t^{\alpha}\right)^{1 / \alpha}} \phi(F(s)) \psi(G(t)) d s d t \\
& \quad \leq D(1,1, x, y ; \alpha)\left(\int_{0}^{x}(x-s)[\phi(f(\sigma))]^{2} d s\right)^{1 / 2}\left(\int_{0}^{y}(y-t)[\psi(g(t))]^{2} d t\right)^{1 / 2},
\end{aligned}
$$

where $D(1,1, x, y ; \alpha)$ is obtained by taking $p=q=1$ in (3.2).

TheOREM 3.5. Let $f, g, p, q, P$, and $Q$ be as in Theorem 3.3, and define $F(s)=$ $1 / P(s) \int_{0}^{s} p(\sigma) f(\sigma) d \sigma$ and $G(t)=1 / Q(t) \int_{0}^{t} q(\tau) g(\tau) d \tau$, for $s \in(0, x), t \in(0, y)$, where $x, y$ are positive real numbers. Let $\phi$ and $\psi$ be as in Theorem 2.5. Then

$$
\begin{aligned}
\int_{0}^{x} \int_{0}^{y} \frac{P(s) Q(s) \phi(F(s)) \psi(G(t))}{\left(s^{\alpha}+t^{\alpha}\right)^{1 / \alpha}} d s d t \\
\leq D(1,1, x, y ; \alpha)\left(\int_{0}^{x}(x-s)[p(s) \phi(f(s))]^{2} d s\right)^{1 / 2} \\
\quad \times\left(\int_{0}^{y}(y-t)[q(t) \psi(g(t))]^{2} d t\right)^{1 / 2}
\end{aligned}
$$

where $D(1,1, x, y ; \alpha)$ is obtained by taking $p=q=1$ in (3.2).

The proofs of Theorems 3.4 and 3.5 can be completed by following the proof of Theorem 3.3 and by closely looking at the proofs of Theorems 2.5 and 2.6 and by making use of the integral versions of Jensen's and the Schwarz inequalities. Here, we omit the details.

\section{REFERENCES}

[1] G. S. Davis and G. M. Peterson, On an inequality of Hardy's (II), Quart. J. Math. Oxford Ser. 15 (1964), 35-40.

[2] M. Gao, On Hilbert's inequality and its applications, J. Math. Anal. Appl. 212 (1997), no. 1, 316-323. MR 98b:26014. Zbl 890.26011.

[3] G. H. Hardy, J. E. Littlewood, and G. Pólya, Inequalities, Cambridge University Press, Cambridge, 1952. MR 13,727e. Zbl 634.26008.

[4] Y.-H. Kim, Refinements and extensions of an inequality, J. Math. Anal. Appl. 245 (2000), no. 2, 628-632. MR 2000m:26025. Zbl 951.26009.

[5] D. S. Mitrinović, Analytic Inequalities, Die Grundlehren der mathematischen Wisenschaften, vol. 165, Springer-Verlag, New York, 1970. MR 43\#448. Zbl 199.38101.

[6] D. S. Mitrinović and J. E. Pečarić, On inequalities of Hilbert and Widder, Proc. Edinburgh Math. Soc. (2) 34 (1991), no. 3, 411-414. MR 92k:26047. Zbl 742.26014.

[7] J. Németh, Generalizations of the Hardy-Littlewood inequality, Acta Sci. Math. (Szeged) 32 (1971), 295-299. MR 47\#2018. Zbl 226.26020. 
[8] B. G. Pachpatte, A note on some series inequalities, Tamkang J. Math. 27 (1996), no. 1, 77-79. MR 97d:26018. Zbl 849.26008.

[9]___ On some new inequalities similar to Hilbert's inequality, J. Math. Anal. Appl. 226 (1998), no. 1, 166-179. MR 99i:26026. Zbl 911.26012.

Young-Ho Kim: Department of Applied Mathematics (or, Brain Korea 21 Project CoRPs), CHANGWON NATIONAL UNIVERSITY, CHANGWON 641-773, KoreA

E-mail address: yhkim@sarim.changwon.ac.kr 


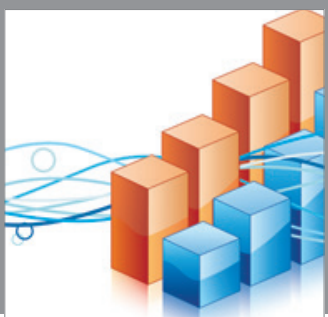

Advances in

Operations Research

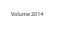

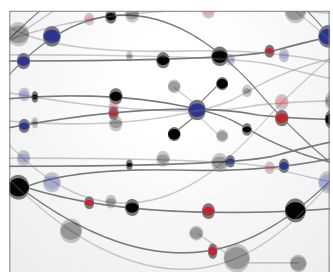

\section{The Scientific} World Journal
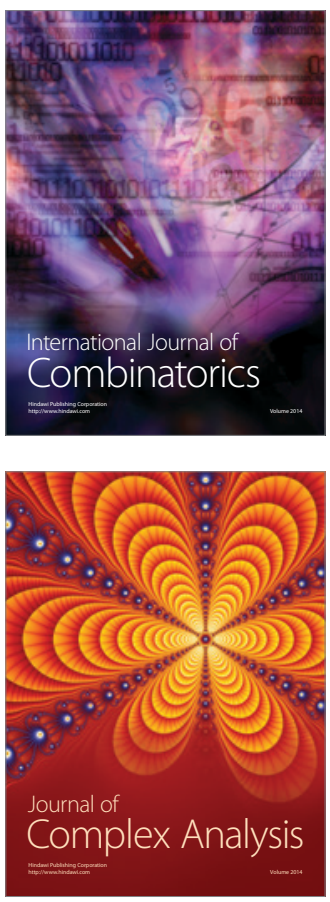

International Journal of

Mathematics and

Mathematical

Sciences
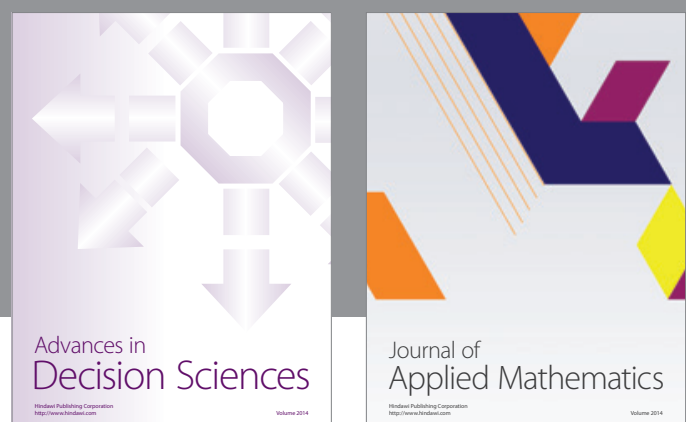

Journal of

Applied Mathematics
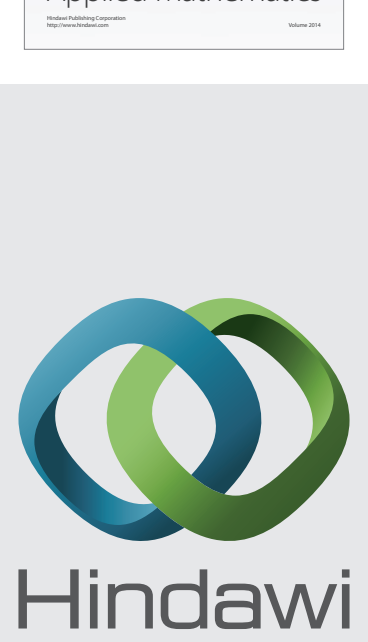

Submit your manuscripts at http://www.hindawi.com
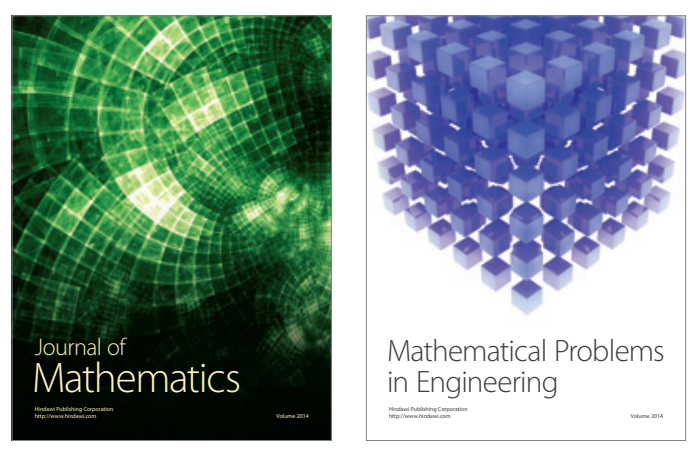

Mathematical Problems in Engineering
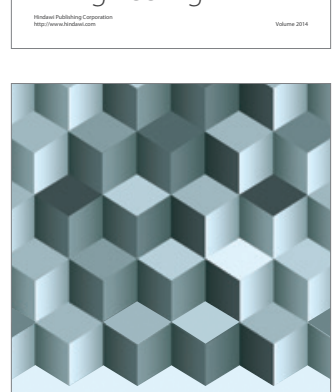

Journal of

Function Spaces
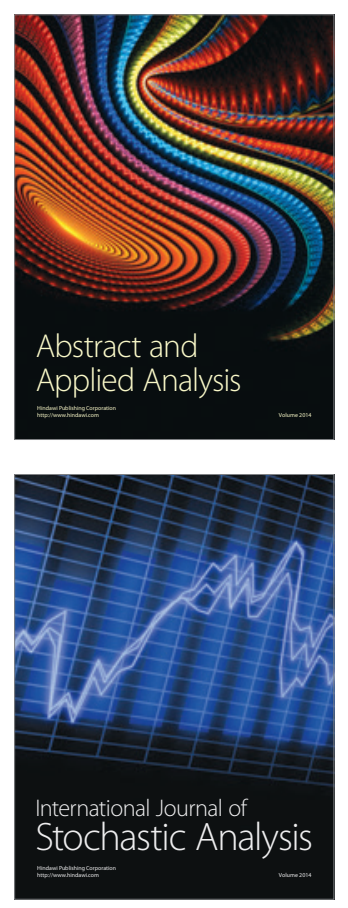

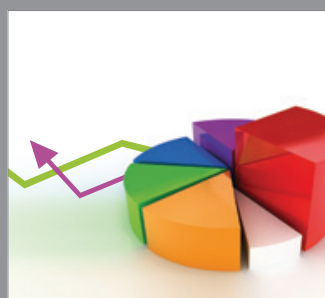

ournal of

Probability and Statistics

Promensencen
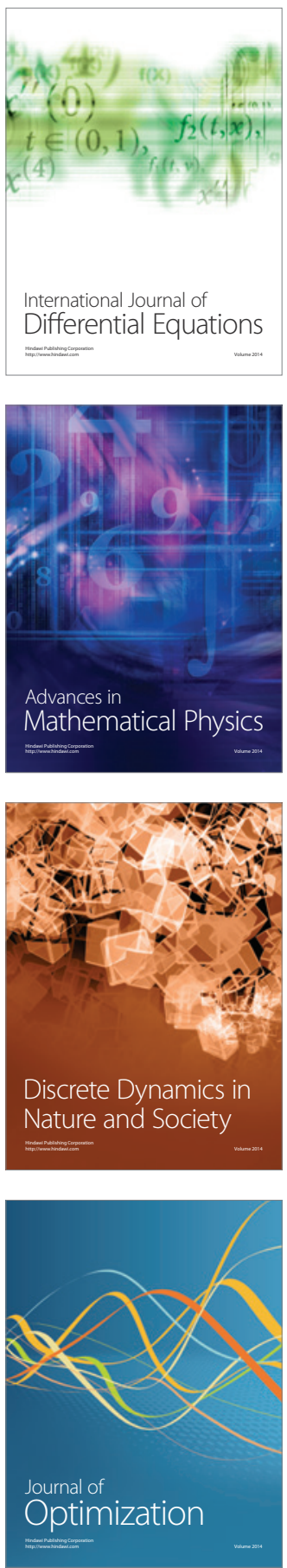